\title{
Injection of harmonics generated in gas in a free-electron laser providing intense and coherent extreme-ultraviolet light
}

\author{
G. LAMBERT ${ }^{1,2,3 *}$, T. HARA ${ }^{2,4}$, D. GARZELLA ${ }^{1}$, T. TANIKAWA ${ }^{2}$, M. LABAT ${ }^{1,3}$, B. CARRE ${ }^{1}$, H. KITAMURA ${ }^{2,4}$, \\ T. SHINTAKE ${ }^{2,4}$, M. BOUGEARD ${ }^{1}$, S. INOUE ${ }^{4}$, Y. TANAKA ${ }^{2,4}$, P. SALIERES ${ }^{1}$, H. MERDJI ${ }^{1}$, 0. CHUBAR ${ }^{3}$, \\ 0. GOBERT ${ }^{1}$, K. TAHARA ${ }^{2}$ AND M.-E. COUPRIE ${ }^{3}$
}

\author{
${ }^{1}$ Service des Photons, Atomes et Molécules, DSM/DRECAM, CEA-Saclay, 91191 Gif-sur-Yvette, France \\ ${ }^{2}$ RIKEN SPring-8 Centre, Harima Institute, 1-1-1, Kouto, Sayo-cho, Sayo-gun, Hyogo 679-5148, Japan \\ ${ }^{3}$ Groupe Magnétisme et Insertion, Synchrotron Soleil, L'Orme des Merisiers, Saint Aubin, 91192 Gif-sur-Yvette, France \\ ${ }^{4}$ XFEL Project Head Office/RIKEN, 1-1-1, Kouto, Sayo-cho, Sayo-gun, Hyogo 679-5148, Japan \\ *e-mail: guillaume.lambert@synchrotron-soleil.fr
}

Conventional synchrotron radiation sources enable the structure of matter to be studied at near-atomic spatial resolution and picosecond temporal resolution. Free-electron lasers promise to extend this down to femtosecond timescales. The process by which free-electron lasers amplify synchrotron light-known as self-amplified spontaneous emission ${ }^{1-3}$-is only partially temporally coherent, but this can be improved by seeding it with an external laser ${ }^{4,5}$. Here we explore the use of seed light produced by high-order harmonic generation in a gas $^{6-9}$, covering wavelengths from the ultraviolet to soft X-rays. Using the SPring-8 Compact SASE Source test accelerator ${ }^{10-12}$, we demonstrate an increase of three orders of magnitude in the intensity of the fundamental radiation at $160 \mathrm{~nm}$, halving of the free-electron laser saturation length, and the generation of nonlinear harmonics ${ }^{13}$ at $54 \mathrm{~nm}$ and $32 \mathrm{~nm}$. The low seed level used in this demonstration suggests that nonlinear harmonic schemes should enable the generation of fully coherent soft $\mathrm{X}$-rays at wavelengths down to the so-called 'water window', vital for the study of biological samples.

In a free-electron laser (FEL), a high-quality relativistic electron beam propagates through an undulator system, composed of two vertical series of alternating magnetic poles, which establish a periodic magnetic field. The horizontally wiggling particles radiate synchrotron radiation-the spontaneous emission (SE), which interacts with the electron beam through energy exchange along the undulator. Then, the electron bunch density is modulated in the longitudinal dimension by a period close to the SE wavelength (bunching), so that the different slices start to emit in phase. In self-amplified spontaneous emission (SASE), the amplification starting from SE requires long undulators to reach saturation. Moreover, the bunching takes place independently in different parts of the bunch and limits the temporal coherence of the radiation. Hence, seeding an FEL with an external coherent source forces a faster and more efficient bunching of the electrons than in SASE. The saturation is then achieved over shorter undulator length, resulting in a more compact source. Because amplification is coherently triggered within the seed pulse length, instead of erratically from SE in the SASE, the pulse temporal/spectral profiles should no longer present statistical fluctuations ${ }^{1,2}$ (spikes). Similarly, seeding should eliminate the temporal jitter, that is, the random structure of the FEL pulse, typically varying in the femtosecond range. Furthermore, the FEL spectral gain width, which is inversely proportional to the number of undulator periods, becomes large enough at short wavelengths to match and amplify the broadband seed while preserving its coherence properties, so that ultrashort pulses in the femtosecond range can in principle be produced in the vacuum-ultraviolet to X-ray region.

Recently, injection of a single-pass FEL by the third laser harmonic of a Ti:sapphire laser from crystals $(266 \mathrm{~nm}, 4 \mu \mathrm{J}, 5 \mathrm{ps}$ full-width at half-maximum, FWHM) led to large amplification ${ }^{5}$. Seeding an FEL with high-order laser harmonics generated in gas (high-order harmonic generation, HHG) offers relevant extension to short wavelengths. Indeed, HHG sources with sufficient peak power now exist down to the water window ${ }^{9}$. Thus seeding an FEL becomes pertinent for generating intense fully coherent radiation in this spectral range. The ångström wavelength range could even be reached using the high-gain harmonic-generation (HGHG, ref. 4) scheme, coupled to a cascade configuration ${ }^{14}$. Moreover, the FEL radiation spectrum is tunable in the vacuum-ultravioletextreme-ultraviolet region either by switching from one harmonic to another, by controlling the driving laser pulse in $\mathrm{HHG}^{15-17}$ or/and by chirping the electron beam energy ${ }^{18}$.

A schematic diagram of the combined HHG and FEL setup is given in Fig. 1. A Ti:sapphire laser (100 fs FWHM) is loosely focused on a xenon gas cell, favouring uniform HHG conditions and optimum energy output ${ }^{7,8}$. Odd third to twenty-first harmonics of the fundamental laser frequency are generated, for example the fifth harmonic at $160 \mathrm{~nm}$ with energy per pulse close to $1 \mu \mathrm{J}$ within a $50 \mathrm{fs}$ (FWHM) duration. The HHG seed beam is spectrally selected, refocused, spatially and temporally overlapped with the electron beam $(150 \mathrm{MeV}, 1 \mathrm{ps}$ FWHM) in the two consecutive undulator sections, tuned to the $160 \mathrm{~nm}$ wavelength. The amplification of the vacuum-ultraviolet seed, which is spatially/spectrally characterized on an imaging spectrometer, is 


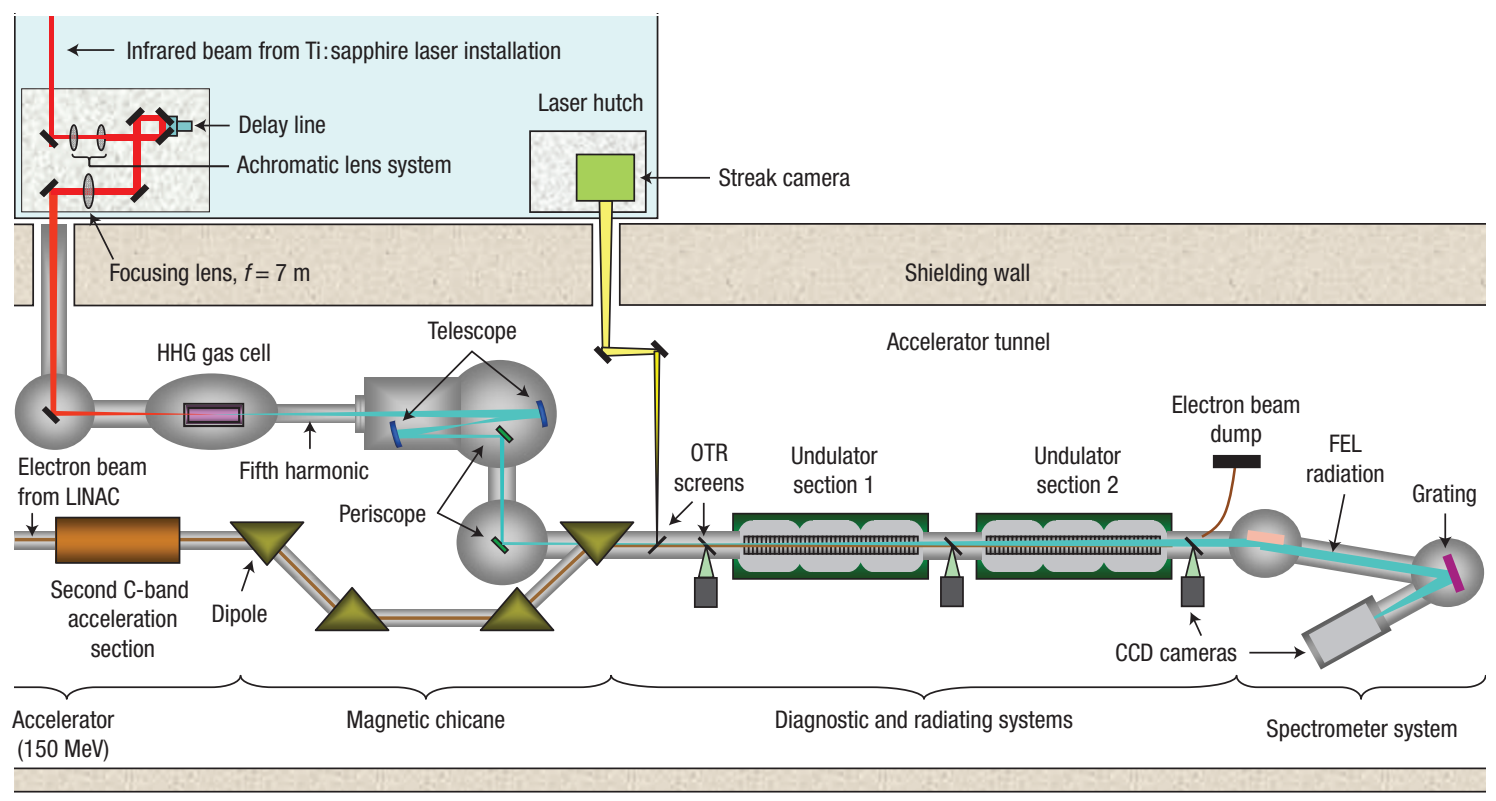

Figure 1 Layout of the experiment. A Ti:sapphire laser $(800 \mathrm{~nm}, 20 \mathrm{~mJ}, 100 \mathrm{fs}$ FWHM, $10 \mathrm{~Hz}$ ) is loosely focused into a xenon gas cell (focal length $f=7 \mathrm{~m})$, optimizing HHG output. Using the telescope and periscope optics ( $\mathrm{CaF}_{2}$ mirrors), the $\mathrm{HHG}$ seed beam is spectrally selected, refocused and spatially and temporally overlapped with the electron beam ( $150 \mathrm{MeV}, 1 \mathrm{ps}$ FWHM, $10 \mathrm{~Hz}$ - LINAC stands for linear accelerator) in the two consecutive undulator sections 1 and 2, which are both tuned to $160 \mathrm{~nm}$, corresponding to the fifth harmonic of the laser. The beam position is monitored on optical transition radiation (OTR) screens.

optimized by varying the time delay between the seed and the electron beam (see the Methods section).

Figure 2 shows typical two-dimensional intensity distributions of the seeded FEL emission (with HHG injection), the unseeded emission (without HHG injection) and the HHG seed, measured with the first undulator section tuned to $160 \mathrm{~nm}$, while the second section was off resonance. The vertical axis corresponds to the spatial position and the horizontal axis to the wavelength. The 'quarter moon' shape, observed in the seeded and unseeded configurations, results from the spectrally shifted off-axis emission of the undulator ${ }^{19}$. Single-shot profiles of the seeded FEL and HHG emissions, cut at the maximum of intensity of the two-dimensional pictures along the spatial and spectral axes, reveal quasi-perfect gaussian profiles. Although the electron-beam parameters are not best tuned and the FEL gain is relatively limited at the time of the experiment, the measured spectra show clear amplification once seeded and even at low seed energy $(0.53 \mathrm{~nJ}$ per pulse). At the centre of the spectral profiles, the amplification factor reaches more than 500 from the seed, and $\sim 2,600$ from the unseeded emission. The nonlinear-harmonic (NLH) intensities are also increased greatly compared with the unseeded NLH emission: the third NLH (factor 312) and the fifth NLH (factor 47) are shown in Fig. 3. The pulse energies have been calibrated with respect to the SE level ${ }^{20}$ (see the Methods section), giving, for the seeded FEL after the first undulator section, about $0.34 \mu \mathrm{J}$ at $161.15 \mathrm{~nm}$ (fundamental), $0.3 \mathrm{~nJ}$ at $53.55 \mathrm{~nm}$ (third harmonic) and $12 \mathrm{pJ}$ at $32.1 \mathrm{~nm}$ (fifth).

As a second step, the first and second sections of the undulator are both tuned to $160 \mathrm{~nm}$ and the energy of the HHG seed is increased from $0.53 \mathrm{~nJ}$ to $4.3 \mathrm{~nJ}$. The FEL pulse energies are measured and shown in Fig. 4 (green and violet symbols) as a function of undulator length. The ratio of the pulse energies between the seeded and unseeded emissions after the first undulator section remains high (a factor of 3,700), but the amplification factor is rather restricted with respect to the HHG seed (a factor of 10). This indicates a slight transverse misalignment (filling factor $\left.{ }^{21} F_{\mathrm{f}}<1\right)$ and spectral detuning $\left(\lambda_{\text {seed }}-\lambda_{\mathrm{SE}} \leq 0\right)$ compared with the previous measurements (red symbols in Fig. 4). Also, the FEL gain was smaller because of the lower electron-beam brightness (electron density in six-dimensional phase space, $B_{\mathrm{p}}$ ). Indeed, because there was no feedback system on the electron beam at the time of the experiment, its brightness and energy showed a slow drift over one day. In the second undulator section, whereas the unseeded emission is still amplified by a factor of 700, the seeded emission is enhanced by a factor of only seven, a sign of saturation (Figs 4,5).

Numerical simulations have been carried out with the GENESIS $^{22}$ code integrated in the SRW environment ${ }^{20}$, and with the PERSEO Time Dependent $\operatorname{code}^{23}$ (PTD), taking into account the spectral overlap and three-dimensional effects, such as the transverse mismatch. The two simulations present quite similar-within $5 \%$ - pulse energy evolution. They predict a large amplification of the seed in the first undulator section and saturation in the second section (green line in Fig. 4). The partial disagreement compared with measurements remains within the incertitude range of the parameters used in the simulations.

The spectral properties of the seeded FEL are of major interest, because seeding has a main aim of improving them. Our study shows that the HHG broadband seed (FWHM relative spectral width: $0.54 \%$ for $0.53 \mathrm{~nJ}, 0.75 \%$ for $4.3 \mathrm{~nJ}$ ) is well adapted to the FEL spectral gain (0.54-0.88\% derived from unseeded emission). Therefore, the HHG seed should be efficiently coupled to the amplifier. After the first undulator section, the spectral profiles of the seeded FEL emission approximately reflect that of the seed, and no random spike structure is generated (Figs 2,3). Indeed, the quasi-gaussian shape indicates that the amplification is driven by the seed.

It is worth noticing that the spectral narrowing is present. The measured relative spectral widths of the seeded FEL are reduced compared with the unseeded one from $0.54 \%$ to $0.46 \%$ $(0.53 \mathrm{~nJ}$ seed $)$ and $0.88 \%$ to $0.44 \%(4.3 \mathrm{~nJ}$ seed $)$. These values 


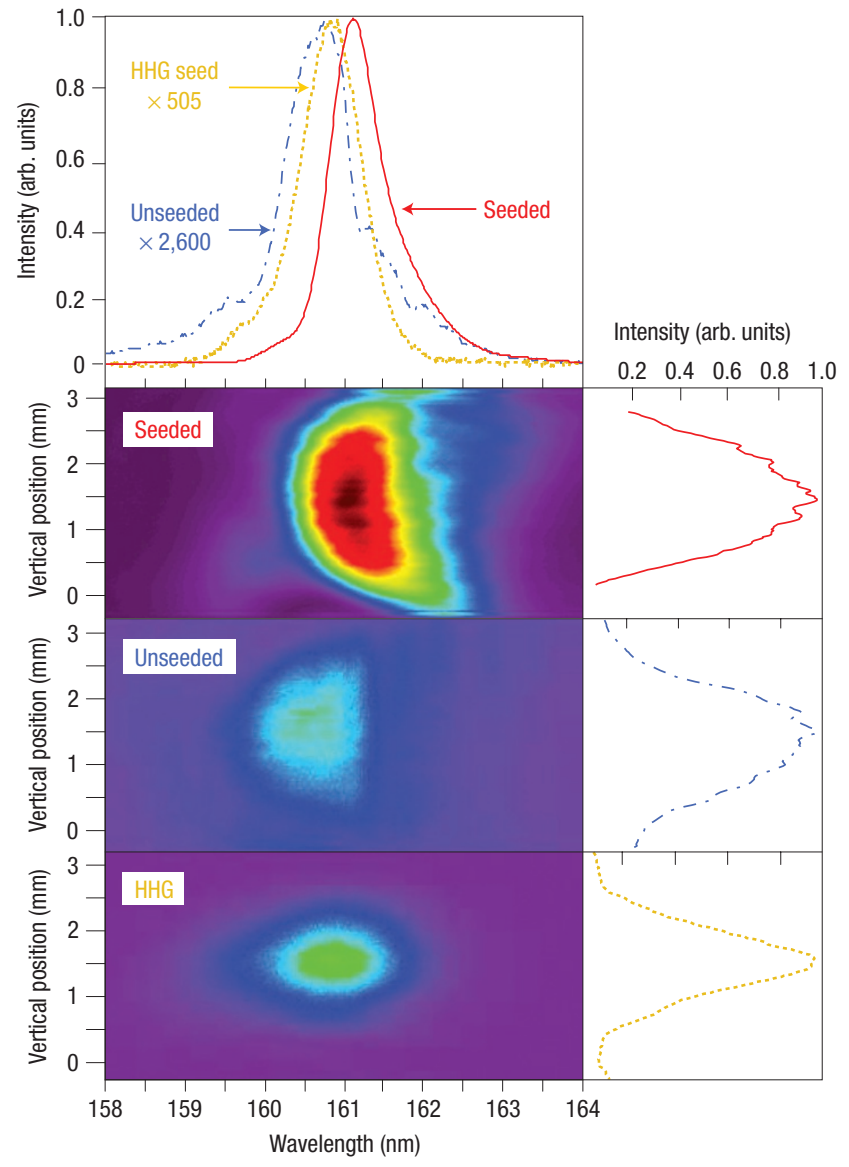

Figure 2 Comparison between the FEL seeded emission, the unseeded emission and the HHG seed at the fundamental wavelength $(160 \mathrm{~nm})$. The spatial (vertical) and spectral distributions are mapped on the CCD (charge-coupled device) camera of a spectrometer; spatial (right) and spectral (up) profiles are plotted at maximum intensity. The lines correspond to the seeded (single shot, line) and unseeded emission (averaged on 10 shots, dash-dot) and the HHG seed (single shot, dots). The seed pulse energy was $0.53 \mathrm{~nJ}$ and only the first undulator section was used for amplifying the HHG pulse.

are in reasonable agreement with the predicted values obtained in the PTD simulations, giving respectively $0.41 \%(0.53 \mathrm{~nJ}$ seed $)$ and $0.40 \%(4.3 \mathrm{~nJ}$ seed). Simultaneously, the relative spectral bandwidths of the measured NLHs are narrowed with the HHG injection from $2.66 \%$ to $0.84 \%$ for the third NLH and $2.54 \%$ to $1.1 \%$ for the fifth NLH.

Though not yet demonstrated experimentally, we can reasonably assume that the phase properties of the seed should be preserved. In particular, for fully coherent seed pulses $^{24}$, the seeded FEL should also show high temporal coherence for the fundamental, that is, have a temporal duration close to the Fourier-transform limit. For the $0.53 \mathrm{~nJ}$ seed case, the fundamental Fourier-transform duration (FWHM), estimated from the measured spectral width, is $57 \mathrm{fs}$ and is in good agreement with the $64 \mathrm{fs}$ value, obtained from the numerical simulations ${ }^{23}$ in our experimental conditions (HHG Fourier-transform duration of $39 \mathrm{fs}$ ). Finally, the peak powers are estimated to be $5.4 \mathrm{MW}, 6.1 \mathrm{~kW}$ and $0.35 \mathrm{~kW}$, respectively, for the fundamental, the third and fifth NLHs, assuming the pulse duration values of the simulations.

In addition to the spectral narrowing, a red-shift is observed on the seeded emission with respect to the unseeded one; this probably
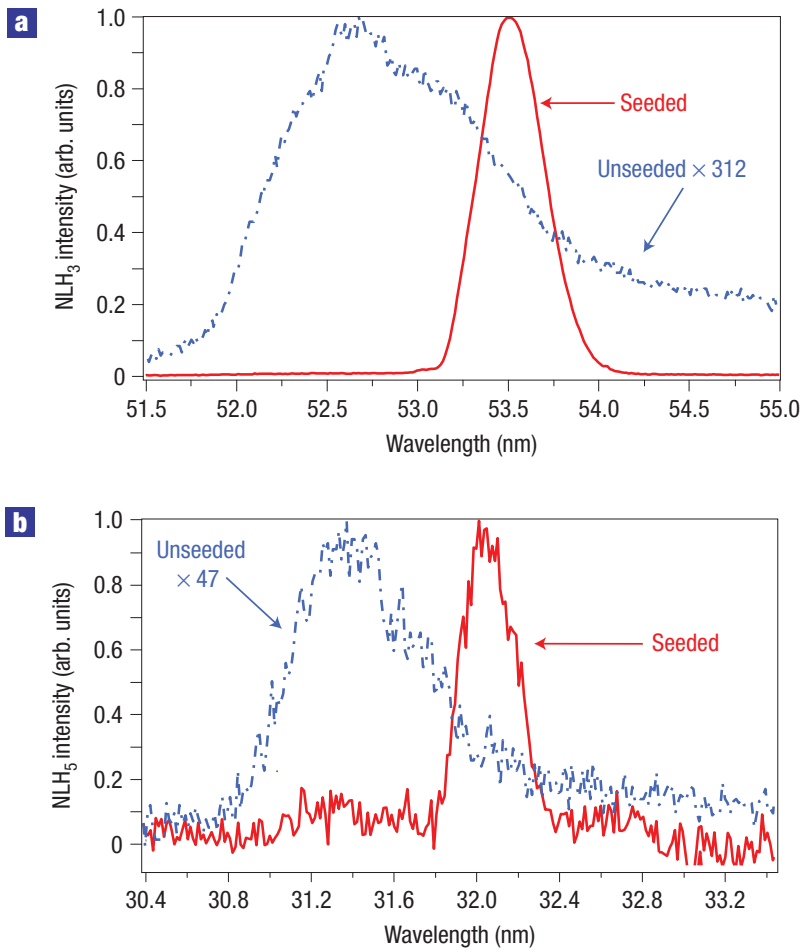

Figure 3 Spectra of the FEL seeded and unseeded emission at the wavelengths of the third and fifth NLHs. The spectra have been obtained by integrating the two-dimensional distributions of the CCD images (as for Fig. 2) over the vertical dimension. The seeded (single shot, line) and unseeded (averaged on 10 shots, dash-dot) FEL emissions are plotted for the third (a) and fifth (b) NLHs. The seed pulse energy was $0.53 \mathrm{~nJ}$ and only the first undulator section was used for radiating the NLHs.

corresponds to a change of the FEL resonance condition due to an energy loss of the electrons and/or to a possible chirp in the beam energy.

Once both undulator segments are set in resonance with the seed wavelength, the spectral distribution shows a sideband structure $^{19}$ (Fig. 5). This probably indicates an overbunching of the electrons carrying out synchrotron oscillations after saturation ${ }^{25}$. The sidebands involve an increase of the amplified FEL bandwidth to $0.67 \%$ ( $0.57 \%$ in PTD simulations), as expected in a postsaturation regime ${ }^{19}$. The spectral red-shift approximately reaches twofold larger values $(\sim 2.5)$ compared with the singleundulator case, which is in good agreement with the simulation ${ }^{23}$ $(\sim 2)$, demonstrating additional energy transfer from the electron beam to the FEL field.

Recently, an HHG seed has been injected in an X-ray laser at $32 \mathrm{~nm}$ operated in a gas-phase plasma ${ }^{26,27}$, delivering pulses of microjoule energy at $10 \mathrm{~Hz}$ repetition rate. On account of the narrow spectral gain, the output pulse duration remains larger than $500 \mathrm{fs}$. Complementarily in our work, the large spectral gain in the FEL device enables the coherent amplification of a broadband seed $\left(\Delta \lambda / \lambda \sim 10^{-3}\right)$, achieving much shorter pulses at typically kilohertz repetition rate.

The available HHG output energy in the $60-30 \mathrm{~nm}$ range is typically a few tens of nanojoules to $100 \mathrm{~nJ}$ per pulse. This proceeds from $\mathrm{HHG}$, for instance, in the 'plateau' region of $\mathrm{Ar}^{6}$, where conversion efficiency reaches $10^{-5}-10^{-6}$, using laser pulses in the $10 \mathrm{~mJ}$ range at kilohertz repetition rate, now becoming standard. These energies are comparable to the seed pulse energy 


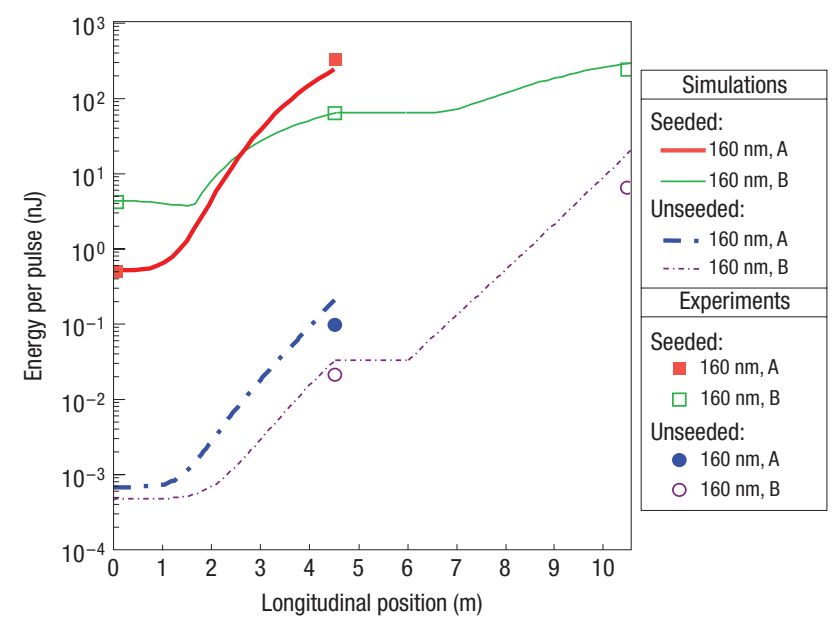

Figure 4 Evolution of the $160 \mathrm{~nm}$ FEL pulse energy along the two undulator sections: comparison of experimental data and simulations using PTD code. The two sections, each of $4.5 \mathrm{~m}$, are separated by a 1.5 -m-long drift space. Two cases of amplification are considered, at low and high HHG seed energy, respectively; they also differ slightly in the electron-beam brightness and the spatial/spectral overlaps between the seed and electron beam.

Case A: $0.53 \mathrm{~nJ}$ seed, $F_{\mathrm{f}}=1, \lambda_{\text {seed }}-\lambda_{\mathrm{SE}}=160.85-160.72=0.13 \mathrm{~nm}$, $B_{\mathrm{p}} \approx 200 \mathrm{~A}(\pi \mathrm{mm} \mathrm{mrad})^{-2}$. Case B: $4.3 \mathrm{~nJ}$ seed, $F_{\mathrm{f}}=0.4$, $\lambda_{\text {seed }}-\lambda_{\mathrm{SE}}=160.84-160.98=-0.14 \mathrm{~nm}, B_{\mathrm{p}} \approx 180 \mathrm{~A}(\pi \mathrm{mm} \mathrm{mrad})^{-2}$. Points are experimental data; lines are calculations for the same conditions.

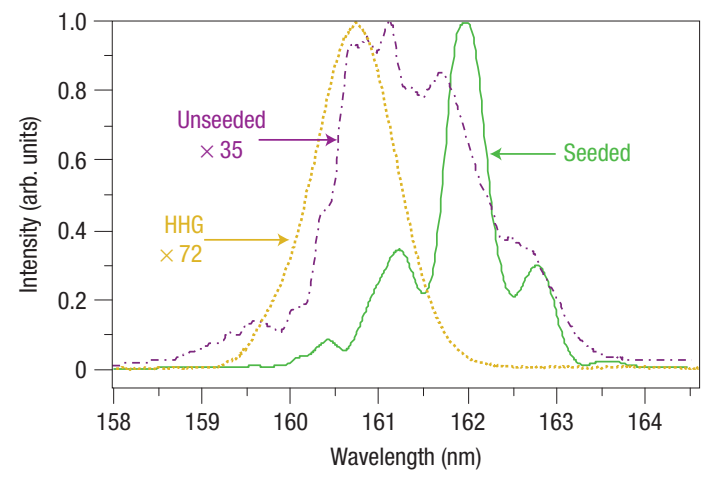

Figure 5 Spectra of the FEL fundamental emission using the two undulator sections: unseeded (single shot, dash-dot) and seeded (single shot, line) obtained with a $4.3 \mathrm{~nJ}$ seed (single shot, dots). The FEL gain is smaller compared with the measurements in Figs 2 and 3 , because of the lower electron-beam brightness $\left(B_{\mathrm{p}}<200 \mathrm{~A}(\pi \mathrm{mm} \mathrm{mrad})^{-2}\right)$, transverse misalignment $\left(F_{\mathrm{f}}<1\right)$ and spectral detuning $\left(\lambda_{\text {seed }}-\lambda_{\text {SE }} \leq 0\right)$.

used in the present experiment. In this range, harmonic radiation shows comparable properties of regular and controllable spatial ${ }^{28}$ and spectral ${ }^{16}$ phases. This allows for the next extension of the amplification and the NLH schemes in a $60-5 \mathrm{~nm}$ range. The water window region can be reached by using a seed at $10 \mathrm{~nm}$ (ref. 8) or even less ${ }^{9}$, generated with $10^{-7}-10^{-8}$ conversion efficiency, that is, at nanojoule energy level in $\mathrm{Ne}$, and for which extreme-ultraviolet multilayer mirrors are efficient. Furthermore, if the HHG seed is combined with the HGHG or HGHG in cascade and NLH coherent emission, the FEL wavelength can be reduced by more than one order of magnitude. Then the ångström range is achievable in a still compact configuration with preservation of the temporal seed coherence.

\section{METHODS}

The SCSS test accelerator is used as a high-brightness electron source $(150 \mathrm{MeV}$, $0.35 \mathrm{nC}, \sim 1 \mathrm{ps} F W H M, 10 \mathrm{~Hz}$ ). It consists of a $500 \mathrm{kV}$ pulsed d.c. electron gun ${ }^{29}$ ( $\mathrm{CeB}_{6}$ thermionic cathode), S-band $(2,856 \mathrm{MHz})$ and $\mathrm{C}$-band $(5,712 \mathrm{MHz})$ acceleration sections ${ }^{10}$. The undulator is composed of two 4.5 -m-long sections, which are in-vacuum type with $15 \mathrm{~mm}$ period and $3 \mathrm{~mm}$ minimum magnetic gap, separated by a $1.5-\mathrm{m}$-long drift space. The harmonic-generation stage is composed of two chambers ${ }^{12}$. In the first one, a Ti:sapphire laser $(800 \mathrm{~nm}$, $20 \mathrm{~mJ}, 100 \mathrm{fs}$ FWHM, $10 \mathrm{~Hz}$ ) is focused with a lens of $7 \mathrm{~m}$ focal length onto a 9-cm-long gas cell permanently filled with xenon to produce the HHG seed radiation, that is, a series of odd harmonics of the fundamental laser frequency. In the second chamber, a telescope composed of two concave $\mathrm{SiC}$ mirrors is used to adjustably focus the seed at the entrance of the first undulator section. The seed beam is aligned in the undulator with two $\mathrm{CaF}_{2}$ mirrors optimized for the fifth harmonic $(160 \mathrm{~nm})$; they completely cut other harmonics and reduce the $800 \mathrm{~nm}$ laser energy in order to avoid the saturation of detectors. The transverse distributions of the electron beam and the residual $800 \mathrm{~nm}$ laser beam, collinear to the HHG beam, are monitored on OTR screens, located at the entrance and exit of each undulator section (Fig. 1). The sub-picosecond synchronization between the electron beam and the seed pulse is achieved by locking the Ti:sapphire oscillator to the highly stable $476 \mathrm{MHz}$ clock of the accelerator. The timing is first adjusted with a few picoseconds resolution using a femtosecond streak camera (Hamamatsu Photonics FESCA-200-C6138), on which the $800 \mathrm{~nm}$ laser light and the OTR emission from the electron beam are injected. Then an optical delay line on the infrared laser path enables a fine adjustment of the synchronization with an accuracy of about $10 \mathrm{fs}$. At the exit of the second undulator section, the radiation is directed into a dispersive spectrometer using a Au-coated $\mathrm{SiO}_{2}$ flat mirror $\left(85^{\circ}\right.$ angle of incidence). The spectrometer incorporates vertical and horizontal slits positioned in front of a concave laminar grating (Shimatsu Corp. Au coating, $N=2,400$ lines $\mathrm{mm}^{-1}, R=922 \mathrm{~mm}$ ) near normal incidence. The detector is a high-sensitivity back-illuminated CCD camera (Princeton Instruments PI*SX $400,1,340 \times 400,20 \times 20 \mu \mathrm{m}$ pixels), covering the $1-10 \mathrm{keV}$ spectral range. As the spectral dispersion is horizontal, the two-dimensional image on the CCD camera combines vertical spatial position (vertical axis) and wavelength (horizontal axis). Finally, the undulator gap is tuned so that the undulator radiation and the seed are spectrally matched in the spectrometer.

To obtain absolute pulse energies, measured intensities are first scaled to the measured SE from the first undulator section, and then calibrated using the calculated absolute SE energy ${ }^{20}$. The measured SE is assumed to be only the fundamental emission. The harmonics of the undulator emission are ignored in the calibration, even if they are also reflected in higher orders of the grating. This may lead to conservative estimates of the pulse energies. The pulse energies of the NLH emission are also derived from the fundamental SE taking into account the spectral response of the optical elements on the radiation path. The electron-beam parameters used in the simulations are $1.45 \times 10^{-3}$ r.m.s. slice energy spread, around $200 \mathrm{~A}(\pi \mathrm{mm} \mathrm{mrad})^{-2}$ brightness and $8 \mathrm{~m}$ averaged beta function ${ }^{30}$. The peak powers are estimated from the pulse durations obtained in PERSEO Time Dependent and GENESIS simulations.

Received 19 September 2007; accepted 28 January 2008; published 9 March 2008.

References

1. Bonifacio, R., Pellegrini, C. \& Narducci, L. M. Collective instabilities and high-gain regime in a free-electron laser. Opt. Commun. 50, 373-378 (1984).

2. Saldin, E. L., Schneidmiller, E. A. \& Yurkov, M. V. Statistical properties of radiation from VUV and X-ray free electron laser. Opt. Commun. 148, 383-403 (1998).

3. Ayvazyan, V. et al. First operation of a free-electron laser generating $\mathrm{GW}$ power radiation at $32 \mathrm{~nm}$ wavelength. Eur. Phys. J. D 37, 297-303 (2006).

4. Yu, L. H. et al. High-gain harmonic-generation free-electron laser. Science 289, 932-934 (2000).

5. Shaftan, T. et al. First SASE and seeded FEL lasing of the NSLS DUV FEL at 266 and $400 \mathrm{~nm}$. Nucl. Instrum. Methods Phys. Sect. A 507, 15-18 (2003).

6. Salieres, P., L'Huillier, A. \& Lewenstein, M. Coherence control of high-order harmonics. Phys. Rev Lett. 74, 3776-3779 (1995).

7. Hergott, J. F. et al. Extreme-ultraviolet high-order harmonic pulses in the microjoule range. Phys. Rev A 66, 021801 (2002).

8. Takahashi, E., Nabekawa, Y. \& Midorikawa, K. Low-divergence coherent soft x-ray source at $13 \mathrm{~nm}$ by high-order harmonics. Appl. Phys. Lett. 84, 4-6 (2004).

9. Seres, J. et al. Source of coherent kiloelectronvolt X-rays. Nature 433, 596 (2005).

10. Shintake, T., Kitamura, H. \& Ishikawa, T. Proc. 2003 SRI Conf., AIP Vol. 705, 117 (2004). 
11. Garzella, D. et al. Using VUV high-order harmonics generated in gas as a seed for single pass FEL. Nucl. Instrum. Methods Phys. Sect. A 528, 502-505 (2004).

12. Lambert, G. et al. Proc. 2005 FEL Conf. (JACOW) 224-227 (2005).

13. Freund, H. P. Nonlinear theory of short-wavelength free-electron lasers. Phys. Rev. E 52 , 5401-5415 (1995).

14. Dattoli, G. \& Ottaviani, P. L. Design considerations for X-ray free electron lasers. J. Appl. Phys. 86, 5331-5336 (1999).

15. Eichmann, H. et al. Polarization-dependent high-order two-color mixing. Phys. Rev. A 51 3414 (1995).

16. Lee, D. G. Coherent control of high-order harmonics with chirped femtosecond laser pulses. Phys. Rev. Lett. 87, 243902 (2001).

17. Mauritsson, J. et al. Measurement and control of the frequency chirp rate of high-order harmonic pulses. Phys. Rev. A 70, 021801(R) (2004).

18. Shaftan, T. \& Yu, L. H. High-gain harmonic generation free-electron laser with variable wavelength Phys. Rev. E 71, 046501 (2005)

19. Sajaev, V. et al. Z-dependent spectral measurements of SASE FEL radiation at APS. Nucl. Instrum. Methods Phys. Sect. A 506, 304-315 (2003).

20. Chubar, O. \& Elleaume, P. Proc. 1998 EPAC Conf. (JACOW) 1177-1179 (1998).

21. Nutarelli, D. et al. Dynamic filling factor in the Super-ACO free- electron laser. Nucl. Instrum. Methods Phys. Sect. A 393, 64-69 (1997).

22. Reiche, S. GENESIS 1.3: A fully 3D time-dependent FEL simulation code. Nucl. Instrum. Methods Phys. Sect. A 429, 243-248 (1999).

23. Giannessi, L. Proc. 2006 FEL Conf. (JACOW) 91-94 (2006).

24. Mairesse, Y. et al. High harmonics XUV spectral phase interferometry for direct electric field reconstruction. Phys. Rev. Lett. 94, 173903 (2005).

25. Huang, Z. \& Kim, K.-J. Transverse and temporal characteristics of a high-gain free-electron laser in the saturation regime. Nucl. Instrum. Methods Phys. Sect. A 483, 504-509 (2002).

26. Zeitoun, P. et al. A high-intensity highly coherent soft X-ray femtosecond laser seeded by a high harmonic beam. Nature 431, 426-429 (2004).
27. Wang, Y. et al. High-brightness injection-seeded soft-x-ray-laser amplifier using a solid target. Phys Rev. Lett. 97, 3901 (2006)

28. Le Déroff, L., Salières, P. \& Carré, B. Beam-quality measurement of a focused high-order harmonic beam. Opt. Lett. 23, 1544-1546 (1998).

29. Togawa, K., Shintake, T., Inagaki, T., Onoe, K. \& Tanaka, T. $\mathrm{CeB}_{6}$ electron gun for low-emittance injector. Phys. Rev. ST Accel. Beams 10, 020703 (2007).

30. Tanaka, H. et al. Proc. 2006 FEL Conf. (JACOW) 769-776 (2006).

\section{Acknowledgements}

We have greatly appreciated contributions from T. Ishikawa, T. Tanaka, H. Tanaka, M. Yabashi, K. Togawa, Y. Otake, T. Ohshima, T. Inagaki, K. Shirasawa and other members of XFEL Project Head Office of RIKEN for their help and support in operating the accelerator and FEL devices. We thank L. Giannessi for his continuous support and for the development of the PERSEO Time Dependent code. We are very grateful to G. Le Chevallier, E. Caprin, J.-F. Hergott, M. Perdrix, P. Monot, P. Monchicourt and P. Breger at CEA-Saclay laser facility, for their technical help on the HHG experiment. We express thanks to the JSPS organization, which offered a grant to G.L. in Japan. Correspondence and requests for materials should be addressed to G.L., T.H. or M.-E.C.

\section{Author contributions}

M.-E.C. and T.H. are respectively in charge of the French-Japanese collaboration. G.L., with assistance from M.-E.C., T.H., D.G. and B.C., designed and installed the experiment, acquired and analysed data and carried out simulations. T.T., M.L., Y.T. and K.T. helped in experiment tests and laser and synchronization system development. M.B. and S.I. contributed to the design. H.K. and T.S. supervised construction and operation of the SCSS test accelerator. P.S., H.M. and O.G. gave crucial information for the development of the HHG experiment. O.C. developed the implementation of the GENESIS time-dependent code in the SRW environment. All the authors discussed the results and contributed to the final manuscript.

Reprints and permission information is available online at http://npg.nature.com/reprintsandpermissions/ 